\title{
Effect of practice on performance of a skilled motor task in patients with Parkinson's disease
}

\author{
P Soliveri, R G Brown, M Jahanshahi, C D Marsden
}

\begin{abstract}
Parkinson's disease leads to a breakdown in the execution of highly practised, skilled movements such as walking and handwriting. The improved execution of skilled movements with practice can be understood as a process of schema learning, the determining of the relevant parameters of the specific movement. The ability of patients with Parkinson's disease and age matched normal control subjects to improve their performance, with practice, on a skilled motor task, doing up buttons, was assessed. The task was assessed on its own and with simultaneous foot tapping. Both groups showed an initial improvement in the task on its own and deterioration in performance when buttoning with foot tapping. The amount of interference, however, decreased with practice, particularly in the patients with a $2 \mathrm{~Hz}$ tapping rate. The results suggest that patients with Parkinson's disease are capable of schema learning but require more practice than control subjects to achieve comparable levels of performance. This may be a reflection of the fundamental motor dysfunction of the disease rather than a specific learning deficit.
\end{abstract}

Of the various motor symptoms associated with Parkinson's disease-namely, tremor, rigidity, akinesia, and postural abnormalityakinesia is thought to reflect most closely the consequences of disturbed striopallidal function underlying the disease. ${ }^{1} \mathrm{~A}$ variety of behavioural techniques have been used to study akinesia-for example, reaction time, ballistic limb movements, pursuit tracking, simple repetitive movements such as finger tapping or hand squeezing, and performance on tests of manual speed and dexterity such as pegboards.

Such tasks, however, bear little relation to the types of difficulties which patients with Parkinson's disease experience in real life. Typically, the laboratory tasks involve simple, arbitrary, unskilled movements. These contrast with the complex highly skilled actions such as walking and handwriting with which Parkinson's disease patients have difficulty. It has been observed clinically that patients can often perform such actions only with conscious effort. Indeed, so striking is this feature of the disease that Marsden has suggested that patients with Parkinson's disease have difficulty in "the automatic execution of learned motor plans." ${ }^{2}$ Before considering this suggestion in more detail, it is necessary to discuss some key concepts.

\section{Automaticity, skill, and motor learning}

Two broad classes of mental processes have been distinguished: those that are performed automatically, and those that require a degree of conscious or voluntary control and draw on the individual's limited processing capacity. This distinction was most clearly formulated in the Two Process theory of Shiffrin and Schneider ${ }^{3}$ and applied to a wide range of cognitive and perceptual tasks. Controlled tasks have been described in the literature as attention or effort demanding, ${ }^{4}$ capacity demanding, ${ }^{5}$ or resource demanding. ${ }^{6}$ Although derived from different models, the terms can be used interchangeably. In all cases, a contrast is drawn with automatic processing not requiring attention, capacity, effort, or resources.

The Two Process theory has undergone a number of revisions in the light of recent evidence. ${ }^{7}$ Firstly, many processes cannot be thought of as either automatic or controlled. Rather, there is more of a continuum with processes being more or less automatic or controlled. Secondly, a particular process or set of processes cannot be thought of as being inherently automatic or controlled. They may be automatic under some conditions and controlled under others. Thirdly, within a given task certain components may be relatively automatic and others may be controlled. Despite these revisions, the basic concept of two broad classes of information processing is still useful in understanding behaviour.

The concepts of automatic and controlled processing have particular application in the study of skilled action. Recognising that the individual had a limited processing capacity, William James remarked, "If an act becomes no easier after being done several times, if the careful direction of consciousness were necessary to its accomplishment on each occasion, it is evident that the whole activity of a lifetime might be confined to one or two deeds." 8 James saw the process of practice as the gradual "liberation of attention." Skill acquisition, therefore, can be thought of as the transfer, with practice, from controlled, attention demanding processes to more automatic and less attention demanding processes.

A number of criteria have been used to define automaticity. ${ }^{79}$ Of these, the most empirically useful is the interference criterion. By definition, a fully automatic process is one 
that operates without capacity (or attention, resources, for instance). This implies that it neither suffers from, nor causes interference to, another process being performed at the same time. Therefore, the degree of automaticity of a task-for example, during the process of skill acquisition-can be determined by measuring its susceptibility to suffer from or cause interference to another task carried out at the same time.

Because of the adoption of interference as the main criterion, dual tasks paradigms have become the main method for assessing automaticity and its corollary, control. In such paradigms, the subject performs the main or primary task both on its own and together with a secondary task. The secondary tasks are chosen according to the level of processing resources that they demand. By manipulation of the resource demands of the secondary task, the level of resource remaining for the primary task can be modified. If the primary task is automatic, it should not matter how much resource is available. Performance should be independent of the resource demands of the secondary task. If, however, the primary task is resource demanding (that is, not automatic) then interference will occur once the resource demands of the two tasks exceed the resource limit.

\section{Schema theory and the generalised motor programme}

As noted above, skill acquisition can be thought of as the transfer from controlled, attention demanding processes to more automatic and less attention demanding processes. However, does it follow that a task, once learned to a high level of skill, is necessarily performed automatically under all circumstances?

In considering this question it is necessary to review the concept of the generalised motor programme, ${ }^{10}$ which, in the terminology of Marsden, ${ }^{12}$ was called the motor plan. This is a motor programme for a particular class of action, stored in an abstract form without the parameters necessary to specify any one particular movement. These subordinate and variable parameters include specific muscles groups, and force. Evidence for such generalised motor programmes can be found in skills such as writing. An individual's writing, although unique, has certain invariant features regardless of whether it is performed by the hand, foot, or mouth. " The "writing" programme itself is seen as being stored in a generalised form. The particular muscle groups employed, the force of activation appropriate to the nature of the writing implement, the surface, etc, may be viewed as the subordinate and variable parameters. In Marsden's terminology these are the subordinate motor programmes within the motor plan. The acquisition of a new skill, therefore, may be seen as the building of a generalised motor programme, which then incorporates task relevant parameters.

When a generalised motor programme exist and a skill has been acquired, it still remains for the individual to determine the physical parameters of the movement under the specific conditions existing at that time. Schmidt refers to this process as schema learning. ${ }^{12}$ When performing a skilled movement in a novel situation, the subject will start with knowledge of the initial conditions and the desired outcome of action. From these, and on the basis of previous experience within the generalised motor programme, the motor schema will specify a set of parameters. On execution of the movement, various types of information will be fed back to the motor schema. These include proprioceptive information from the joints and muscles, exteroceptive feedback (vision, sound), and, finally, information about the final outcome of the movement (knowledge of results). This combined information will update the motor schema, which in turn provides a new and more accurate set of parameters for the generalised motor programme.

Because schema learning involves monitoring both proprioceptive and exteroceptive sensory information, it follows that this stage must make some demands on the attentional capacity of the individual. In other words, performance at this stage should be subject to interference from another concurrent task. The degree of interference will presumably depend on the novelty of the conditions under which the skill is being performed and the degree to which the normal parameters have to be modified. For instance, we rarely have to think about walking because we are able to adapt our existing motor programme with great speed and flexibility depending on whether we are walking on concrete or carpet, with large strides or with small steps while moving forward in a queue. In some circumstances, however, such as walking on ice or across a ploughed field, or stepping on to a moving escalator, it takes more time and more conscious effort to determine and reset the parameters of the motor schema for the action. Consequently we may find that we have to stop our conversation and concentrate on keeping our balance. With practice, however, the new parameters are learned and we can continue our conversation.

Returning to Parkinson's disease, how might schema theory help us understand the deficits in executing skilled action? A reasonable hypothesis is that patients with Parkinson's disease have problems not with the automatic execution of the learned motor plan per se but with the process of schema learning - that is, rapid, flexible adaptation of the parameters according to changing demands.

The aim of the present study was to examine the performance of a skilled motor task by patients with Parkinson's disease in comparison to normal patients, using a dual task paradigm. The first decision was the choice of a skilled task. To be considered highly skilled, the task should have been practised for many years. While actions such as walking and handwriting meet this criteria, they are difficult to quantify. Therefore, a decision was made to assess the time required to do up buttons, a 
motor action impaired in patients with Parkinson's disease. ${ }^{13}$ This skill, involving the sequential, bimanual coordination of fingers and thumbs, is acquired in childhood and practised throughout life, often without visual control. That the fine coordination involved in this action is relatively automatic can be judged from the fact that we can normally button a garment at the same time as performing other tasks in everyday life. Whenever a new garment is first encountered, the process of schema learning will involve determining the specific characteristics of the task: button size, size of hole, stiffness of material, etc. Improvement in performance with practice may be taken as evidence that schema learning is taking place. The degree to which learning is controlled or attention demanding may be assessed by the degree to which performance, at different stages, is sensitive to interference from a concurrent secondary task.

\section{Method \\ Subjects}

Twenty one patients (14 men, 7 women) with idiopathic Parkinson's disease and 23 age matched normal controls (9 men, 14 women) participated in the study. The average ages of the groups were, respectively, 60.9 (SD 11.7) years and $62.6(9.2)$ years. The mean age of onset of Parkinson's disease was $51.9(11.5)$ years, giving an average duration of illness of $9.0(6.8)$ years. The patients were assessed on the Hoehn and Yahr scale ${ }^{14}$ and the King's College Hospital (KCH) Parkinson's disease rating scale (scores range from 0 to 117 ). ${ }^{15}$ Three patients were in Hoehn and Yahr stage I, three in stage II, and 15 in stage III. Mean (SD) $\mathrm{KCH}$ score was $27 \cdot 8$ (13.1). Twenty of the patients were taking levodopa (mean (SD) daily dose 675 (292) $\mathrm{mg}$ ), seven in combination with anticholinergics. Eighteen of the patients and 21 of the controls were right handed.

\section{Experimental tasks}

The primary motor task selected was doing up buttons on a cardigan. Two identical woollen cardigans were used, one male version and one female version (buttons on right and left sides respectively). Each cardigan had five buttons of $2.5 \mathrm{~cm}$ diameter. The cardigans were large enough to fit all subjects comfortably. The time taken to do up buttons was the dependent variable, which was measured from the command to start to the successful completion of the last button. The primary motor task of doing up buttons was assessed on its own and with a secondary task. Two levels of secondary task were used: foot tapping at the rates of $2 \mathrm{~Hz}$ and $4 \mathrm{~Hz}$. Foot tapping was monitored using a counter activated by a foot switch. The mean frequency of foot tapping was calculated from the total number of foot taps over the period from start to finish of the primary task.

\section{Procedure}

All subjects performed each of the test condi-
Table 1 Order of test conditions

\begin{tabular}{lcl}
\hline & Trial & \\
& No & Condition \\
\hline Block 1: & $1-4$ & Buttons alone (4 trials) \\
& 5 & Buttons + tapping (2 Hz) \\
& 6 & Buttons + tapping (4 Hz) \\
& $7^{\star}$ & Buttons + tapping + metronome $(2 \mathrm{~Hz})$ \\
& $8^{\star}$ & Buttons + tapping + metronome (4 Hz) \\
& $9^{\star}$ & Buttons + metronome (2 Hz) \\
& $10^{\star}$ & Buttons + metronome (4 Hz) \\
Break & & \\
Block 2: & 11 & Buttons alone \\
& $12^{\star}$ & Buttons + metronome (4 Hz) \\
& $13^{\star}$ & Buttons + metronome (2 Hz) \\
& $14^{\star}$ & Buttons + tapping + metronome $(4 \mathrm{~Hz})$ \\
& $15^{\star}$ & Buttons + tapping + metronome $(2 \mathrm{~Hz})$ \\
& 16 & Buttons + tapping (4 Hz) \\
& 17 & Buttons + tapping (2 Hz) \\
18 & Buttons alone \\
\hline
\end{tabular}

ऋ Data not presented.

tions in a fixed order (table 1). First, the subjects were given four trials of buttoning alone. They were then tested on buttoning with foot tapping, first at $2 \mathrm{~Hz}$ and then at $4 \mathrm{~Hz}$. There then followed four more trials of buttoning. Two of the trials were performed with foot tapping but with the addition of a metronome set to the appropriate target tapping frequency $(2 \mathrm{~Hz}$ or $4 \mathrm{~Hz})$. The two other trials were buttoning alone but with the metronome and were designed to assess another issue relating to the value of external pacing stimuli on task performance; as such they were secondary to the main question being addressed by this paper. In fact, the metronome had no significant impact on performance in either group. The data from these trials will not be reported here. However, it is important to bear in mind that these trials provided opportunities for extra buttoning practice, both without and with a secondary task.

After this first block of trials the subjects had a five minute break. After the break the subjects had a further trial of buttoning alone, followed by the above conditions in reverse order, including four more trials with a metronome; these data will not be reported here. This second block of trials ended with a final trial of buttoning alone.

\section{Statistical analysis}

Time for buttoning alone was moderately skewed in both groups. Analysis of buttoning time without a secondary task was based on square root transformed data. Buttoning with a secondary task was highly skewed, particularly in the patient group, but the degree of skewness varied in the different conditions. As a result, no single transformation could adequately normalise all of the data. Because of this, non-parametric analyses were performed on the dual task data, and the results are reported in terms of medians and interquartile ranges (the range containing the middle $50 \%$ of the distribution).

\section{Results}

The two groups did not differ in terms of age $(t=0.54, \mathrm{df}=42, \mathrm{p}=0.59)$, handedness 


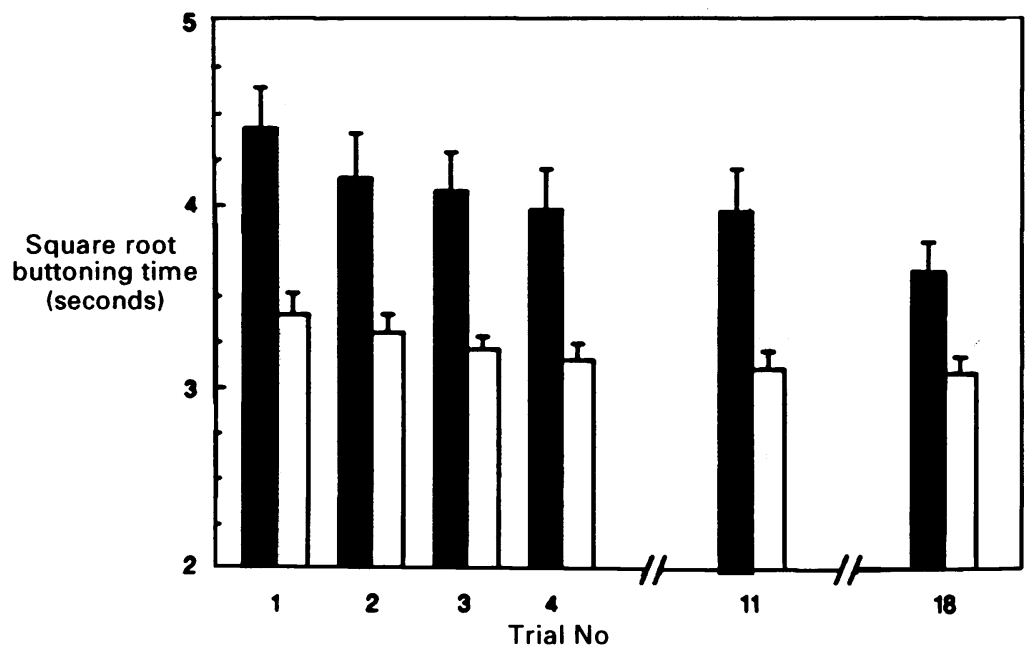

Figure 1 Mean (and standard error) square root buttoning time (seconds) for six trials of buttoning alone. $\square$ Parkinson's disease group; $\square$ Control group

$\left(\chi^{2}=0.34, \mathrm{df}=1, \mathrm{p}=0.56\right)$ or sex ratio $\left(\chi^{2}=2 \cdot 32, \mathrm{df}=1, \mathrm{p}=0 \cdot 13\right)$.

CHANGES IN PERFORMANCE ON BUTTONING ALONE Figure 1 shows the mean square root transformed times for the trials of buttoning alone 1-4 11 18. Analysis of variance showed that, overall, the patients were significantly slower at buttoning than the control group $(F(1,42)=$ $15 \cdot 7, \mathrm{p}<0.001)$. There was a significant main effect of trial number $(F(5,38)=15 \cdot 4, \mathrm{p}<$ $0.001)$, and a significant group $\times$ trial interaction $(F(5,38)=3 \cdot 23, \mathrm{p}<0 \cdot 05)$. To explore this interaction term further, a series of post hoc analyses were performed. First, the data for the initial four practice trials were considered. Although, as before, the main effects for group

Table 2 Buttoning times (seconds) with secondary tasks (foot tapping)

\begin{tabular}{|c|c|c|c|c|c|}
\hline \multirow[b]{2}{*}{ Tapping frequency } & \multirow[b]{2}{*}{ Trial No } & \multicolumn{2}{|c|}{ Parkinson's disease patients } & \multicolumn{2}{|l|}{ Control } \\
\hline & & Median & $I Q R$ & Median & $I Q R$ \\
\hline $\begin{array}{l}2 \mathrm{~Hz} \\
2 \mathrm{~Hz} \\
4 \mathrm{~Hz} \\
4 \mathrm{~Hz}\end{array}$ & $\begin{array}{r}5 \\
17 \\
6 \\
16\end{array}$ & $\begin{array}{l}19.0 \\
16.4 \\
18.5 \\
17.5\end{array}$ & $\begin{array}{l}13 \cdot 6-26 \cdot 6 \\
12 \cdot 4-21 \cdot 6 \\
14 \cdot 1-25 \cdot 1 \\
13 \cdot 1-21 \cdot 0\end{array}$ & $\begin{array}{r}11.1 \\
11.0 \\
11.3 \\
9.8\end{array}$ & $\begin{array}{l}9 \cdot 3-13 \cdot 8 \\
8 \cdot 3-13 \cdot 4 \\
8 \cdot 9-13 \cdot 7 \\
8 \cdot 4-12 \cdot 2\end{array}$ \\
\hline
\end{tabular}

IQR = Interquartile range.

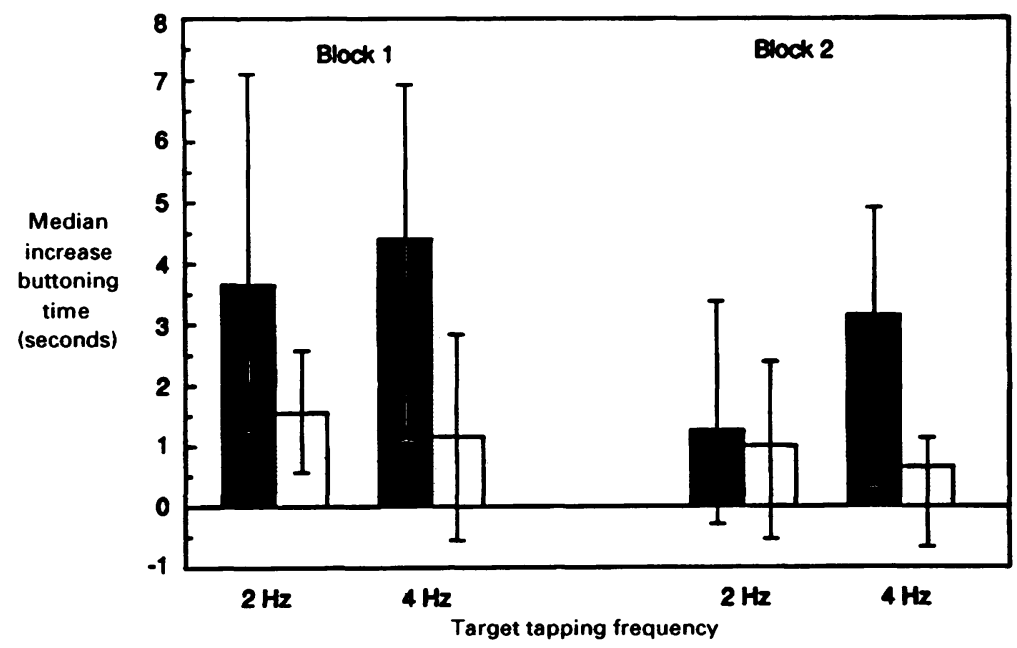

Figure 2 Median (and interquartile range) increase in buttoning time with foot tapping at $2 \mathrm{~Hz}$ and $4 \mathrm{~Hz}$ target frequencies for two test blocks. Parkinson's disease group; $\square$ Control group and trial were significant, there was no significant interaction $(F(3,40)=0.92, \mathrm{p}=0.44)$. Change in performance with initial practice, therefore, was the same in both groups. This implies that the significant group $\times$ trial interaction when all six trials of buttoning alone were considered was due to differential changes between the two groups in the last two trials. As can be seen from figure 1 , the patients showed further improvement between trials 11 and $18(t=2.99, \mathrm{df}=20, \mathrm{p}<0.01)$ while the control group did not $(t=0 \cdot 28, \mathrm{df}=22, \mathrm{p}=$ $0 \cdot 78$ ). To obtain a baseline measure of buttoning time for assessing dual task interference effects, the mean of trials 4,11 , and 18 (buttoning alone) was calculated for each subject. The median baseline score for the patients was 13.5 (interquartile range 11.3-19.7) seconds and for the controls 9.7 $(7 \cdot 7-11 \cdot 6)$ seconds.

DUAL TASK PERFORMANCE

Buttoning performance

Patients with Parkinson's disease were slower than controls to complete the buttoning task under all dual task conditions in both blocks of trials (table 2). To test whether the tapping secondary tasks had any interfering effects on the primary task, the baseline buttoning time was compared with buttoning performance with each of the secondary tasks (MannWhitney $U$ tests). The difference in buttoning time was significant $(p<0.05)$ for the patients for both $2 \mathrm{~Hz}$ and $4 \mathrm{~Hz}$ rates in both blocks. The controls also showed a significant increase in buttoning time with the two tapping rates when first assessed (trials 5 and 6), but in the second block (trails 16 and 17) the slowing was significant only with the $2 \mathrm{~Hz}$ tapping rate.

To assess the amount of interference, difference scores were calculated for each subject under each condition by subtracting the time for buttoning alone from the time for buttoning with foot tapping. The median difference scores are shown in figure 2 . The patients showed a greater slowing $(p<0.05)$ with both the $2 \mathrm{~Hz}$ and $4 \mathrm{~Hz}$ tapping rate for the first test (trials 5 and 6), but for the subsequent tests (trials 16 and 17) the difference between the two groups was significant $(p<0.05)$ only for the $4 \mathrm{~Hz}$ rate. At the slower rate of tapping, the increase in buttoning time was the same in the two groups.

When the increase in buttoning time was considered as a percentage of baseline performance rather than as an absolute difference, the increase at the $2 \mathrm{~Hz}$ rate for the patients did not differ $(p>0.10)$ from that of the controls on either the first trial (median $23.8 \%$ patients $v 17.4 \%$ controls) or the second trial $(10.5 \% v$ $9 \cdot 3 \%$ ). For the $4 \mathrm{~Hz}$ rates, the patients showed a greater proportional slowing $(p<0.05)$ on the first trial $(36.7 \% v 13.3 \%)$ while on the second trial the difference only approached significance $(17 \cdot 3 \% v 6 \cdot 7 \% ; \mathrm{p}<0 \cdot 10)$.

\section{Foot tapping performance}

Performance on the secondary tasks in conjunction with the buttoning task are shown in Table 3. Analysis of variance was performed on 
Table 3 Secondary task performance (foot tapping rate)

\begin{tabular}{|c|c|c|c|c|c|}
\hline \multirow{2}{*}{$\begin{array}{l}\text { Target tapping } \\
\text { frequency }\end{array}$} & \multirow[b]{2}{*}{ Trial No } & \multicolumn{2}{|c|}{ Parkinson's disease patients } & \multicolumn{2}{|l|}{ Controls } \\
\hline & & Mean rate & $S D$ & Mean rate & $S D$ \\
\hline $\begin{array}{l}2 \mathrm{~Hz} \\
2 \mathrm{~Hz} \\
4 \mathrm{~Hz} \\
4 \mathrm{~Hz}\end{array}$ & $\begin{array}{r}5 \\
17 \\
6 \\
16\end{array}$ & $\begin{array}{l}1 \cdot 8 \\
2 \cdot 1 \\
2 \cdot 9 \\
3 \cdot 3\end{array}$ & $\begin{array}{l}0 \cdot 4 \\
0 \cdot 4 \\
0 \cdot 8 \\
0 \cdot 9\end{array}$ & $\begin{array}{l}1.9 \\
2.2 \\
3.8 \\
3.8\end{array}$ & $\begin{array}{l}0.3 \\
0.3 \\
0.7 \\
0.7\end{array}$ \\
\hline
\end{tabular}

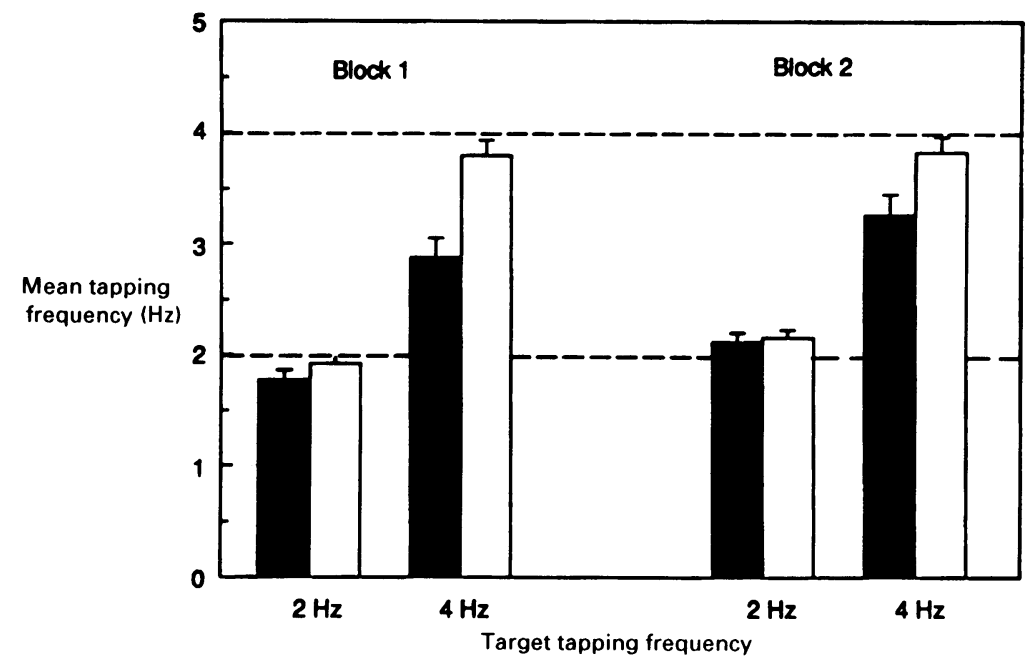

Figure 3 Mean (and standard error) foot tapping rates at target frequencies of $2 \mathrm{~Hz}$ and $4 \mathrm{~Hz}$ for two test blocks $\square$ Parkinson's disease group; $\square$ Control group

the mean tapping rate for the two frequencies $(2 \mathrm{~Hz}$ and $4 \mathrm{~Hz}$ ) for the two trials at each frequency. The data are shown in figure 3. Overall, the patients tapped at a slower rate than the control subjects $(F(1,42)=9 \cdot 75, \mathrm{p}<$ $0 \cdot 01)$. There was also a significant main effects of test trial (first $v$ second test block) $(F(1,42)$ $=20 \cdot 15, \mathrm{p}<0.001$ ) with subjects tending to tap at a faster rate on the second trial for both target frequencies. Significant interactions were found for group $\times$ tapping rate $(F(1,42)$ $=12.94, \mathrm{p}<0.01)$ and group $\times$ test trial $(F(1,42)=4 \cdot 13, \mathrm{p}<0.05)$. The three way interaction (group $\times$ tapping rate $\times$ test trial) was not significant. To investigate these interactions a series of post hoc paired comparisons were performed to assess the between group and within subject effects. These showed no significant difference between the tapping rates of the two groups when the target frequency was $2 \mathrm{~Hz}$ (trials 5 and 17). The patients, however, tapped at a significantly lower rate when the target frequency was $4 \mathrm{~Hz}$, in both the first test block (trial 6) $(t=4 \cdot 15, \mathrm{df}=42, \mathrm{p}<$ $0.001)$ and second (trial 16) $(t=2 \cdot 49, \mathrm{df}=42$, $\mathrm{p}<0.05)$. Across the two blocks at each frequency, both the patients $(t=3 \cdot 68, \mathrm{df}=20$, $\mathrm{p}<0.01)$ and controls $(t=3.37, \mathrm{df}=22, \mathrm{p}<$ 0.01 ) increased their tapping rate for the $2 \mathrm{~Hz}$ condition, while only the patients increased the rate of tapping where the target frequency was $4 \mathrm{~Hz}(t=2 \cdot 88, \mathrm{df}=20, \mathrm{p}<0.01)$.

ASSOCIATION BETWEEN TASK PERFORMANCE AND CLINICAL MEASURES

Both primary (buttoning) and secondary (foot tapping) performances were related to a range of clinical measures in the patient group. Consistently significant correlations $(r>0.46$, $\mathrm{p}<0.05)$ were found with total $\mathrm{KCH}$ score.
Of the subscales, primary and secondary task performances were most strongly $(\mathrm{p}<0.05)$ and consistently related to speech, tremor, ability to rise from a chair, ability to make alternating hand movements, tapping with the right foot, and a summed disability score. Inconsistent or weak associations were found with autonomic function, posture, stability, and rigidity. Performance across measures was also unrelated ( $p<0.05)$ to duration of illness or levodopa dose. Increase in buttoning time with foot tapping compared to buttoning alone was unrelated to any clinical variable.

The seven patients taking anticholinergic medication were compared with those taking only dopa drugs. The two groups did not differ ( $p>0.05$ ) on any of the clinical or performance variables.

\section{Discussion}

The aim of the present experiment was to investigate the ability of patients with Parkinson's disease to learn the task parameters necessary to carry out a skilled action involving a generalised motor programme or plan, in this case buttoning. Two aspects of the results were considered: improvement in buttoning speed with practice, and task performance during foot tapping. The results are discussed in relation to a possible slowness in schema learning in Parkinson's disease and to the possible influence of the fundamental motor deficits of the disorder on performance change with practice.

IMPROVEMENT IN BUTTONING SPEED WITH PRACTICE Patients and normal controls showed a similar decrease in buttoning time over the first four trials of buttoning alone (fig 1), despite different starting levels of performance. Assuming that buttoning was already a highly skilled action, this can be seen as evidence for schema learning in both groups, and to a similar degree.

By the same argument, subsequent improvement in performance, with practice, would suggest that continued learning was taking place. For buttoning alone, no further change was shown for the control group after the initial trials, but the patients showed an additional improvement in performance by the end of the experiment. This might suggest that schema learning was taking place at a slower rate in the patients than in the controls. There are problems, however, with this interpretation of the data. Change in buttoning performance with practice as a measure of learning has the potential problem of ceiling effects-that is, with the physical constraints of the task it may be difficult for subjects to show any significant speed improvements beyond a certain limit, even if learning is continuing to take place. Thus, the failure of the control subjects to show any decrease in buttoning time beyond trial 4 need not imply that learning had reached its limit. Similarly, the patients may have shown continued improvement because they started with a poorer level of performance and therefore had further to go before reaching 
their ceiling. The poorer initial level of the patients can be understood simply in terms of the motor deficit of the disease, as suggested by the association between buttoning performance and aspects of the motor symptomatology such as tremor, which correlated highly with buttoning speed.

Therefore, from the data on buttoning alone, we can conclude that schema learning, as indicated by the improvement in performance with practice, occurs in Parkinson's disease, and that initial learning happens at the same rate as in control subjects. The worse initial level of the patients, however, may mean that they continue improving for longer (although never to the level of controls) before the ceiling effects of the task are reached.

DUAL TASK PERFORMANCE

Change in performance on the buttoning task alone was only one criterion chosen for assessing schema learning. Separate evidence may be found in the degree to which the secondary task, foot tapping, interfered with buttoning speed. If the control subjects had determined the parameters of the action and buttoning was fully automatic, foot tapping should have had little impact on performance. However, despite reaching a stable level of buttoning performance after four trials, controls still showed significant interference effects on the subsequent initial trials (trials 5 and 6) of buttoning with foot tapping. Later, however, after more practice on the buttoning task, the degree of dual task interference had decreased (trials 16 and 17) and was no longer significant in the case of the $4 \mathrm{~Hz}$ tapping frequency. This decrease in the interference effect suggests that schema learning was still taking place after trial 4 , offering support to the suggestion above that buttoning performance was subject to ceiling effects. One might assume that, had the control subjects been given more practice, the interference effects might have disappeared completely.

What of the patients? As shown in figure 2, the initial interference effects were significantly greater than in the control group. With practice, however, the level of interference decreased, to the point where it was no greater than in the controls, at least for the $2 \mathrm{~Hz}$ frequency. With the $4 \mathrm{~Hz}$ rate significant interference effects were still observed. So, in the patients, as in the controls, continuous schema learning seemed to be taking place throughout the repeated buttoning practice.

As before, however, there are problems with a simple interpretation of the results. These relate to the performance of the two groups on the secondary task, foot tapping. Control subjects were able to tap at rates close to the two target frequencies. It is clear from figure 3, however, that the patients had considerable difficulty with the $4 \mathrm{~Hz}$ tapping rate. This does not simply reflect the patients tapping more slowly. Qualitatively, their tapping tended to consist of periods of fast tapping and then sudden "freezing" and erratic performance before normal rhythmical performance was resumed. It is doubtful, therefore, whether the
$4 \mathrm{~Hz}$ foot tapping was suitable for use as a secondary task to compare interference effects in the two groups. The greater interference shown by the patients could reflect either that less schema learning had taken place for the buttoning or that foot tapping was more difficult. Fortunately, the performance of the patients on the $2 \mathrm{~Hz}$ task was both quantitatively and qualitatively similar to that of the controls, with relatively even, unbroken, rhythmical movements. It is still possible that despite comparable levels of performance at the slower rate the patients still found the task more demanding than normal subjects and thus produced more interference with the buttoning task. This seems unlikely, however, given the fact that by the second trial with $2 \mathrm{~Hz}$ tapping the interference in the patients did not differ significantly for that of the control group.

The data from the dual task trials lead us to similar conclusions to those derived from the buttoning performance. The decrease in secondary task interference supports the contention that schema learning was continuing to take place in both groups. Whether the initially greater interference produced by $2 \mathrm{~Hz}$ tapping in the patient group was due to less schema learning is difficult to determine. More important, perhaps, is the fact that by the end of practice the interference effect was no greater than that shown by controls, suggesting that the degree of learning and the level of automaticity of the buttoning task was similar in the two groups.

Although the decrease in interference is consistent with an improvement in schema learning, there are other processes by which buttoning performance might improve. One possiblity is that the apparent improvement in performance was due to a change in strategy, trading off performance on the two simultaneous tasks-in the second set of dual task trials, the subjects may have concentrated solely on the buttoning task, ignoring the tapping task. If this were the case we would have expected tapping performance to have deteriorated in the second block of trials while buttoning performance improved. In fact, the speed of the patients' tapping increased for both the 2 $\mathrm{Hz}$ rate and the $4 \mathrm{~Hz}$ rate. Thus, at the same time as the patient group's buttoning speed was increasing, the tapping rate was also increasing. There is no evidence, therefore, for any change in the trade off between the primary and secondary tasks. Another possibility is that the subjects were not learning the buttoning schema but that they were learning a completely new skill, in which the two tasks were combined into a superordinate task. If this was indeed the case then the evidence suggests that such a process is relatively normal in patients with Parkinson's disease.

The initial hypothesis was that patients with Parkinson's disease have problems with schema learning and that this may contribute to the problems that they experience with skilled actions in everyday life, particularly in relatively novel situations. To what degree do the present data support this hypothesis? Firstly 
and most importantly, the patients seemed to show clear evidence of schema learning, as indicated both by improved performance on the primary task and by decreased dual task interference with practice, at least for the $2 \mathrm{~Hz}$ rate. By the latter index the eventual degree of learning was the same as for the controls. If the patients did have an impairment it was in the amount of practice required to establish and utilise the relevant task parameters. However, as discussed, we should be cautious in drawing any conclusion about slower learning in the patient group, because of problems in equating the level of difficulty of the interfering task (foot tapping), and the initial level of buttoning performance in the two groups. The motor performance of patients with Parkinson's disease is fundamentally slow and hypometric, as evidenced by even the most simple, repetitive, and unskilled task. Either as a reflection of or due to this basic deficit, the initial buttoning parameters set by the patients were likely to be less accurate than those of the controls. The patients were thus starting from a higher baseline and might require more practice to establish an accurate set of parameters. Such basic akinesia might impose a limit on the success of performance, even after schema learning is complete. It is likely that even if they were given unlimited practice the performance of patients with Parkinson's disease would never match that of normal subjects on speeded skilled tasks. This would explain why even highly practiced skills such as walking and handwriting are still abnormal in Parkinson's disease.

We may conclude, therefore, that schema learning is possible in patients with Parkinson's disease but that improvement in performance with practice takes place more slowly. This slowness, however, may be a reflection of the limits imposed by a fundamentally abnormal motor system, rather than to any deficit in learning per se.

A final unanswered question is the degree to which the present results can be generalised to patients not receiving drug treatment. What would be the effect of dopamine withdrawal on schema learning? The only relevant data from the present study is that no aspect of task performance, including the degree of interference, was related to level of levodopa medication. However, a definitive answer could be obtained only by a direct comparison of the medicated and unmedicated states. However, while there might be a slowing in the rate at which performance improved with practice in the untreated state, this would be in the context of generally worsened motor function. Thus the same problems present in comparing patients with controls would apply when two patient groups, one medicated and the other unmedicated, were compared.

The financial assistance of the Wellcome Trust (MJ) and the European Science Foundation (PS) is gratefully acknowledged.

1 Marsden CD. Motor disorders in basal ganglia disease. Human Neurobiol 1984;2:245-50.

2 Marsden CD. The mysterious motor function of the basal

3 Shiffin RM, Schneider W. Controlled and automatic human information processing. II. Perceptual learning, automatic attending and a general theory. Psych Rev 1977;84:127-90.

4 Kahneman D. Attention and effort. Englewood Cliffs, NJ: Prentice-Hall, 1973.

5 Morray N. Where is capacity limited? A survey and a model. Acta Psychologia 1967;27:84-92.

6 Norman DA, Bobrow DG. On data-limited and resourcelimited processes. Cognitive Psychol 1975;7:44-64.

7 Neumann $O$. Automatic processing: a review of recent findings and a plea for an old theory. In: Printz W, Sanders AF, eds. Cognition and motor processes. Berlin, Heidelburg: Springer-Verlag, 1984.

8 James W. Principles of psychology Vol 1. New York: Henry Holt, 1890. (Reprinted New York: Dover Publications, 1950).

9 Jonides J, Naveh-Benjamin M, Palmer J. Assessing automaticity. Acta Psychologia 1985;60:157-71.

10 Schmidt RA. Control processes in motor skills. Exercise and Sports Sciences Reviervs 1976;4:229-61.

11 Merton PA. How we control the contraction of our muscles. Sci Am 1972;226:30-7.

12 Schmidt RA. A schema theory of discrete motor skill learning. Psych Rev 1975;82:225-60.

13 Brumlick J, Bosches B. The mechanism of bradykinesia in parkinsonism. Neurology (Minneapolis) 1966;16:337-44.

14 Hoehn MM, Yahr MD. Parkinsonism: onset, progression and mortality. Neurology (Minneapolis) 1967;17:427-42.

15 Parkes JD. Adverse effects of antiparkinsonian drugs. Drugs 1981;21:341-52. 